\title{
TCF19 enhances cell proliferation in hepatocellular carcinoma by activating the ATK/FOXO1 signaling pathway
}

\author{
C. X. ZENG ${ }^{1, *}$, S. B. FU' ${ }^{2, *}$, W. S. FENG ${ }^{1}$, J. Y. ZHAO', F. X. LI' ${ }^{1}$, P. GAO ${ }^{1, *}$ \\ ${ }^{1}$ Department of General Surgery, Guangdong Second Provincial General Hospital, Guangzhou, China; ${ }^{2}$ The Third Affiliated Hospital of Sun \\ Yat-sen University, Guangzhou, China \\ ${ }^{*}$ Correspondence: $z x c q 12333 @ 126 . c o m$ \\ \#Contributed equally to this work.
}

Received December 27, 2017 / Accepted May 21, 2018

\begin{abstract}
Hepatocellular carcinoma (HCC) is one of the most lethal malignancies because of its complexity, high metastasis, recurrence and limited treatment options. Reports state that transcription factor 19 (TCF19) is related to the susceptibility to chronic HBV infection and that it strongly increases the risk of HCC occurrence, but its molecular mechanisms remain unknown. This study analyzed the datasets and confirmed that TCF19 is significantly increased in HCC cell lines and tissues. MTT and colony formation assay revealed that TCF19 over-expression enhances cell proliferation and tumorigenesis. Flow cytometry assay then determined that TCF over-expression helps HCC cell G1/S phase transition, and further research showed that TCF19 up-regulation inhibits $\mathrm{p} 57^{\text {Kip2 }}$, p2 $1^{\text {Cip1 }}$ and $\mathrm{p} 27^{\text {Kip1 }}$ cell cycle suppressors, enhances the expression of cyclin D1 expression and simulates retinoblastoma (Rb), FOXO1 and AKT phosphorylation. In addition, AKT and FOXO1 inhibitors suppress the TCF19 effect on cell proliferation. This demonstrates that AKT/FOXO1 signaling is essential for TCF19 influence on HCC progression, and our combined results suggest that crucial links between TCF19 and HCC can provide a novel target for hepatocellular carcinoma treatment.
\end{abstract}

Key words: hepatocellular carcinoma, TCF19, FOXO1, AKT

Liver cancer is the sixth most common malignant cancer, and the third leading cause of cancer death. There were 745,500 deaths in 2012 and 782,500 new cases are diagnosed globally, and China alone accounts for over $50 \%$ of the total cases $[1,2]$. Hepatocellular carcinoma (HCC) is the most common liver cancer and approximately $90 \%$ of primary liver cancer [3]. In addition, HCC is one of the most lethal malignancies due to its complexities, high metastasis and recurrence and limited treatment options [4].

It has been documented that the fundamental trait of cancer is interrupted cell cycle homeostasis and sustained proliferation [5]. The cell cycle of mitosis is composed of four phases: G1, S, G2 and S phase. Through the four phases, cells duplicate their genetic material and divide into two daughter cells [6]. The progression of the cell cycle is regulated by the cyclin/cyclin-dependent kinase (CDKs) complex. The cyclin D-CDK4/6 complex takes center role in G1/S transition and its binding activates CDKs which then phosphorylate retinoblastoma (RB); a major suppressor protein of G1/S transition $[7,8]$.

Phosphorylation of RB then releases E2Fs which transactivate the target genes in DNA synthesis to induce cell cycle progression [9]. Other genes then regulate the G1/S transition. For example, $\mathrm{p} 21^{\mathrm{CIP} 1}$, $\mathrm{p} 27^{\mathrm{KIP} 1}$ and $\mathrm{p} 57^{\mathrm{KIP} 2}$, recognized as CDK inhibitors (CDKIs), bind to cyclin D-CDK4/6 complex to arrest the cell cycle $[10,11]$, and abnormal expression of any of the above components can induce cancer. Clarifying cell cycle mechanisms is therefore fundamental in curing cancer.

Transcription factor 19 (TCF19) was first identified as a trans-activating factor expressed at the late G1/S boundary of the cell cycle [12]. Genome-wide association studies (GWASs) have illustrated that TCF19 is related to susceptibility to chronic HBV infection and strongly increases the risk of HCC occurrence $[13,14]$. However, the underlying molecular mechanism of TCF19 is poorly understood, so our research investigates the role of TCF19 in HCC and elucidates this mechanism.

Herein, we found that TCF19 expression is increased in HCC, and its over-expression enhances cell proliferation and tumorigenesis by hyper-activating cell cycle progression. This shows that TCF19 is important for cell proliferation and that it can be a novel target in hepatocellular carcinoma cure. 


\section{Materials and methods}

Cell lines. The immortalized normal hepatocellular cell (LO2) and human HCC cell lines (MHCC87H, MHCC97L, Hep3B, HCC-LY5, HepG2, QGY-7701, BEL-7402 and Hub7) were cultured using Dulbecco's modified eagle's medium (DMEM; Invitrogen, Carlsbad, CA, USA) supplemented with $10 \%$ fatal bovine serum (FBS; HyClone, Logan, UT, USA) and $1 \%$ penicillin/streptomycin (HyClone, Logan, UT, USA).

Tissue specimens. Two normal liver tissue specimens and eight HCC tissue specimens were collected in Guangdong Second Provincial General Hospital from 2015 to 2017. For research purposes, we obtained patient informed consent and approval from the Institutional Ethics Committee before collecting specimens. After surgery, the tissues were snap-frozen in liquid nitrogen and stored at $-80^{\circ} \mathrm{C}$ until use.

Plasmids. To over-express TCF19, full-length human TCF19 cDNA was amplified by PCR and then cloned into pMSCV plasmid. For depletion of TCF19, two human TCF19-targeting siRNA sequences were cloned into the plasmid of pSuper-retro-puro, respectively, and primers used are as follows: RNA\#1: CCCAACGCAATCGGAGGAAAT; RNA\#2: ACACTGATCCTAAACTCCATA. Plasmids were then transfected by Lipofectamine 3000 (Invitrogen, Carlsbad, CA, USA).

Real-time PCR (RT-PCR). Total RNA was isolated using Trizol reagent (Invitrogen, Carlsbad, CA, USA). cDNA was amplified using ABI 7500 Fast System (Applied Biosystems, Rockville, MD, USA) and $\alpha$-Tubulin gene is used as reference. The relative expressions of genes were calculated by the equation: $2^{-[(C \text { to f gene)-(Ct of a-tubulin)] }}$, where $\mathrm{Ct}$ represents threshold cycle. The primers of RT-PCR are as follows: TCF19, forward 5'-TCAGCCTGGAAGACCACAGCAG-3' and reverse 5'-CCAAAGGTCAGGAGGTCTCCAT-3'; p57 ${ }^{\text {KIP2}}$, forward 5'-AGATCAGCGCCTGAGAAGTCGT-3' and reverse 5'-TCGGGGCTCTTTGGGCTCTAAA-3'; p21 ${ }^{\mathrm{CIP} 1}$, forward 5'-AGGTGGACCTGGAGACTCTCAG-3' and reverse 5'-TCCTCTTGGAGAAGATCAGCCG-3'; p27 ${ }^{\mathrm{KIP} 1}$, forward 5'-CGTCCTCCATAGCAGCCAAGAT-3' and reverse 5'-ACCCAATGGAGCCCAGGATGAA-3'; Cyclin D1, forward 5'-TCTACACCGACAACTCCATCCG-3' and reverse 5'-TCTGGCATTTTGGAGAGGAAGTG-3'.

Western blotting assay. The protein was subjected to western blotting according to the methods described [15]. Equal total protein amounts were loaded $(30 \mu \mathrm{g} /$ well $)$ and separated by $10.5 \%$ polyacrylamide gels. The primary antibodies were: anti-TCF19, anti-p57, anti-p21, anti-27, anti-E2F1, anti-p-Rb, anti-Rb, anti-Cyclin D1, anti-FOXO1, anti-p-FOXO1, anti-AKT, anti-p-AKT and anti- $\alpha$-Tubulin (diluted 1:2500, Abcam, Cambridge, MA, USA). All membranes were incubated by HRP-conjugated secondary antibody (diluted 1:1500, Abcam, Cambridge, MA, USA), with $\alpha$-Tubulin the loading control.
MTT (3-(4, 5-Dimethyl-2-thiazolyl)2, 5-diphenyl-2Htetrazolium bromide) assay. $2 \times 10^{3}$ cells were seeded in 96-well plates and cells were dyed with MTT reagent for four hours at the indicated time, $(0.5 \mathrm{mg} / \mathrm{ml}$, sigma, St Louis, $\mathrm{MO}$, USA) for 4 hours. $150 \mu \mathrm{l}$ DMSO was then added to the well and absorbance was measured at $490 \mathrm{~nm}$.

Colony formation assay. $5 \times 10^{2}$ cells were placed in 6-well plates and maintained for 10 days. The colonies were fixed with $10 \%$ formaldehyde for 5 minutes and then stained by $1 \%$ crystal violet for 30 seconds.

Flow cytometry. The flow cytometry assay was performed according to the methods previously described [16]. The cell cycle was assayed by Cytomics FC 500 (Beckman Coulter, CA, USA) with CXP software and the percentage of cells in different cell cycle phases was analyzed by modifit LT 3.1 trial cell cycle software.

Animal studies. For xenograft experiments, the BALB/c nude mice were randomly divided into four groups, and each mouse was injected subcutaneously. The tumor volume was measured with digital calipers and calculated by the equation: (length $\times$ width $\left.^{2}\right) / 2$. On 31st day, the mice were euthanized and tumors were excised and weighed.

Immunohistochemistry (IHC). IHC staining assay was carried out on the paraffin-embedded tissues using TCF19 and p-AKT antibodies. IHC staining was quantitatively assayed with the AxioVision Rel.4.6 computerized image analysis system assisted with the automatic measurement program (Carl Zeiss, Oberkochen, Germany). The stained sections were detected at $200 \times$ magnification.

Statistical analysis. All experiments were repeated independently three times and data is presented as mean \pm SD. Statistical analysis was carried out by the SPSS 20.0, and statistical significance by student's $t$ test. The $p<0.05$ was statistically significant.

\section{Results}

TCF19 is over-expressed in HCC tissues. Analysis of data from The Cancer Genome Atlas (TCGA), revealed that the expression of TCF19 is higher in HCC tissues compared to the normal liver tissues (Normal: $n=50$, HCC: $\mathrm{n}=354 ; \mathrm{p}<0.001$; Figure $1 \mathrm{~A}$ ). We then assayed the expression of TCF19 in HCC tissues and corresponding adjacent normal tissue (ANT), and this demonstrated that its expression is increased significantly in HCC tissues compared to corresponding ANT $(n=50 ; p<0.001$; Figure 1B). We further verified the results in the GEO database (GSE25097), and analysis showed that the expression of TCF19 progressively increases in the following order; normal tissue, ANT and the HCC tissue (Normal: $n=5$, ANT: $n=243$, HCC: $n=268$; Figure 1C).

Subsequently, we checked the results by RT-PCR and Western blot, using the cell lines and fresh tissues. The relative expression of TCF19 is dramatically elevated in HCC cell lines (MHCC87H, MHCC97L, Hep3B, HCC-LY5, 

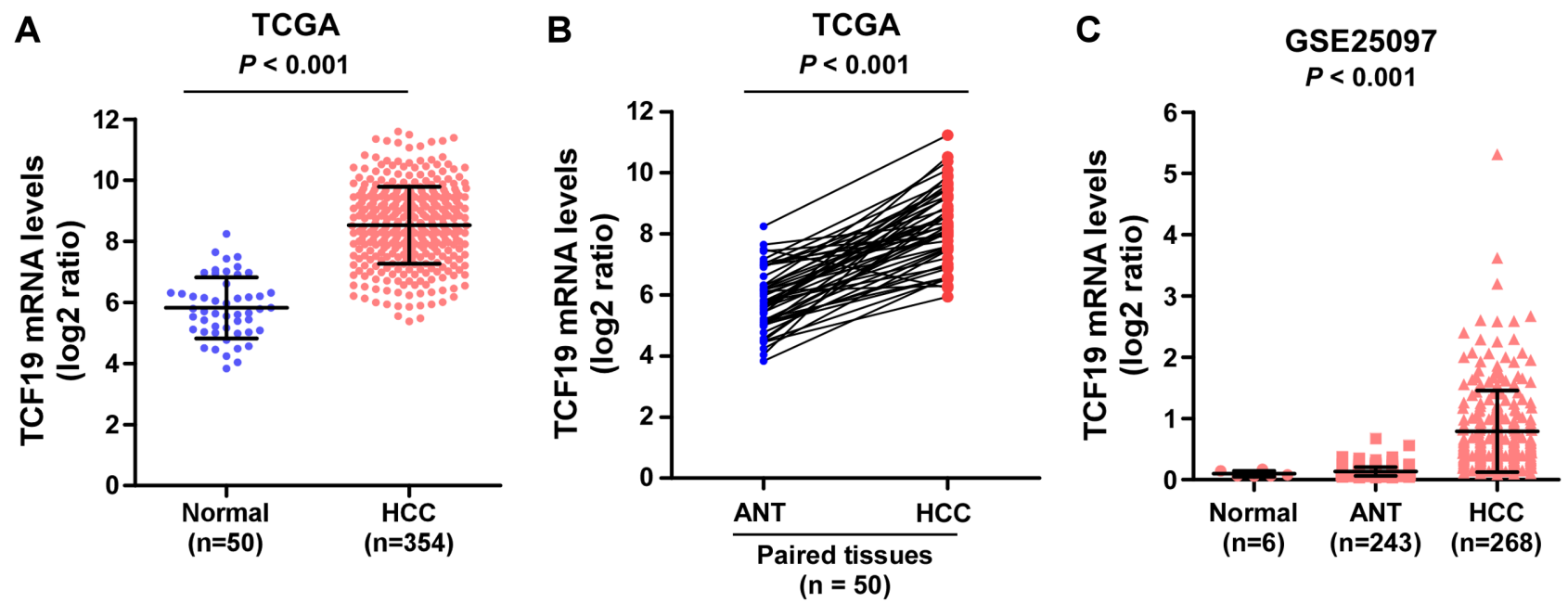

Figure 1. The expression of TCF19 is upregulated in HCC by analyzing datasets. A) Expression profiles of TCF19 in normal liver tissues (n=50) and HCC tissues ( $n=354)(p<0.001$; TCGA). B) The expression of TCF19 in 50 paired HCC tissues (HCC) and adjacent normal tissues (ANT) ( $<<0.001$; TCGA). C) The level of TCF19 in normal liver tissues $(n=6)$, adjacent normal tissues $(n=243)$ and HCC tissues $(n=268)(p<0.001$; GSE25097).

A

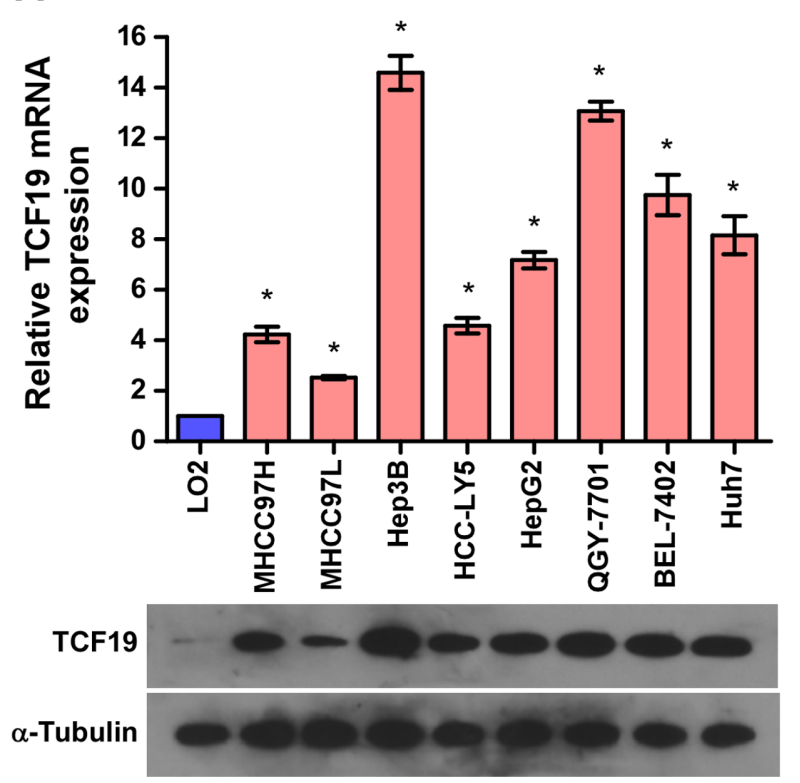

B

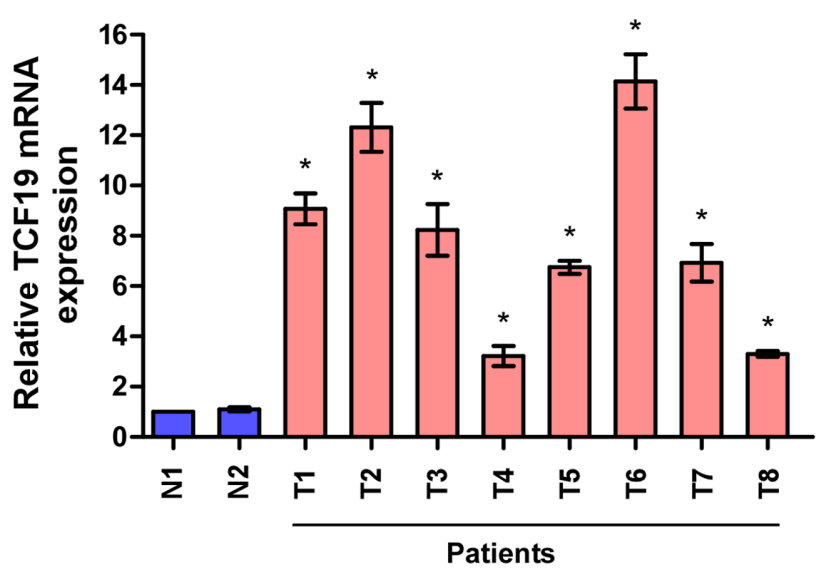

TCF19

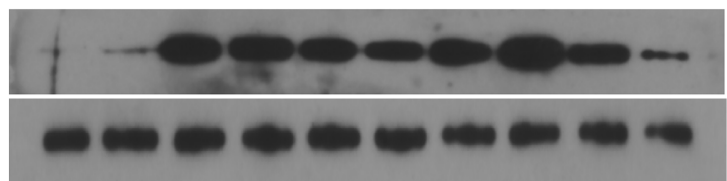

Figure 2. Relative expression of TCF19 in cell lines and tissues. A) Relative expression of TCF19 in immortalized normal hepatocellular cell line (LO2) and eight HCC cell lines (MHCC87H, MHCC97L, Hep3B, HCC-LY5, HepG2, QGY-7701, BEL-7402 and Hub7) by RT-PCR (upper panel) and western blotting (lower panel). B) Relative expression of TCF19 in normal liver tissues (N1 and N2) and HCC tissues (T1-T8) by RT-PCR (upper panel) and western blotting (lower panel). The mRNA level of TCF19 was normalized to that of $\alpha$-Tubulin. And in western blotting assay, $\alpha$-Tubulin served as the loading control.

HepG2, QGY-7701, BEL-7402 and Hub7) compared to the normal liver cell line (LO2) (Figure 2A), and expression in eight HCC tissue specimens was also increased compared to the two normal liver tissue specimens (Figure 2B).

TCF expression is therefore significantly increased in HCC cell lines and tissues compared to both normal liver cell line and tissues.
Over-expression of TCF19 enhances HCC cell proliferation. It has been documented that TCF19 over-expression enhances the cell proliferation in insulinoma cells [17]. So we further explored whether TCF19 has the same impact on HCC cell proliferation by selecting the HepG2 cell which moderately expresses TCF19 for gain-and-loss-of-function assay. Firstly, we screened the stable cells where TCF19 is 
over-expressed and silenced (Figure 3A), and the MTT assay illustrated that over-expression of TCF19 enhances cell proliferation rate and down-regulation inhibits it (Figure 3B). We further verified the results by colony formation assays, and these showed that the over-expression of TCF19 significantly increases colony number but down-regulation reduces it (Figure 3C).
We then examined if TCF19 promotes HCC cell tumorigenesis in vivo. As shown in Figure 3D, TCF19-transduced tumors grew faster than in the controls at each indicated time point, whereas the those formed by TCF19-silenced cells grew much slower than controls. While tumors formed by TCF19-transduction are larger than vector control tumors, those formed by TCF19-silenced cells are smaller than the
A

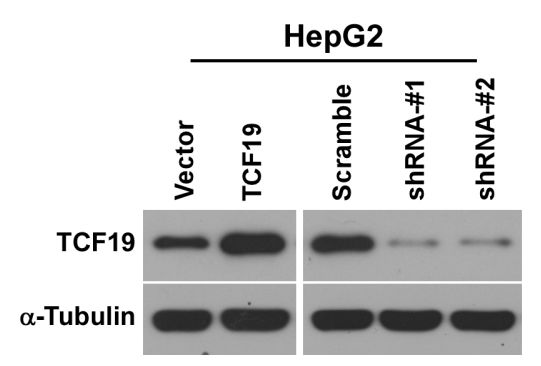

B

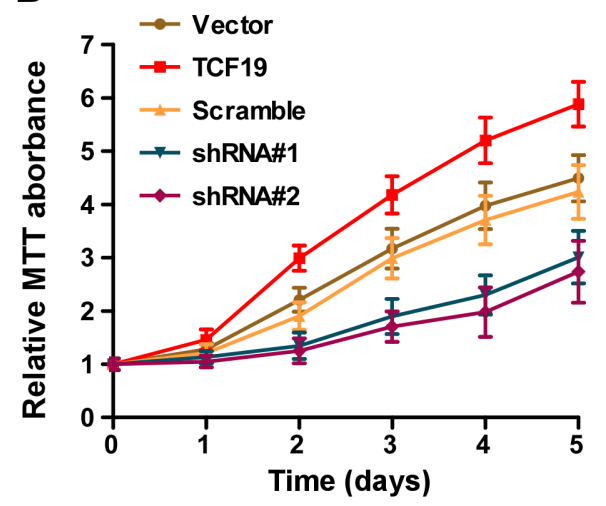

C
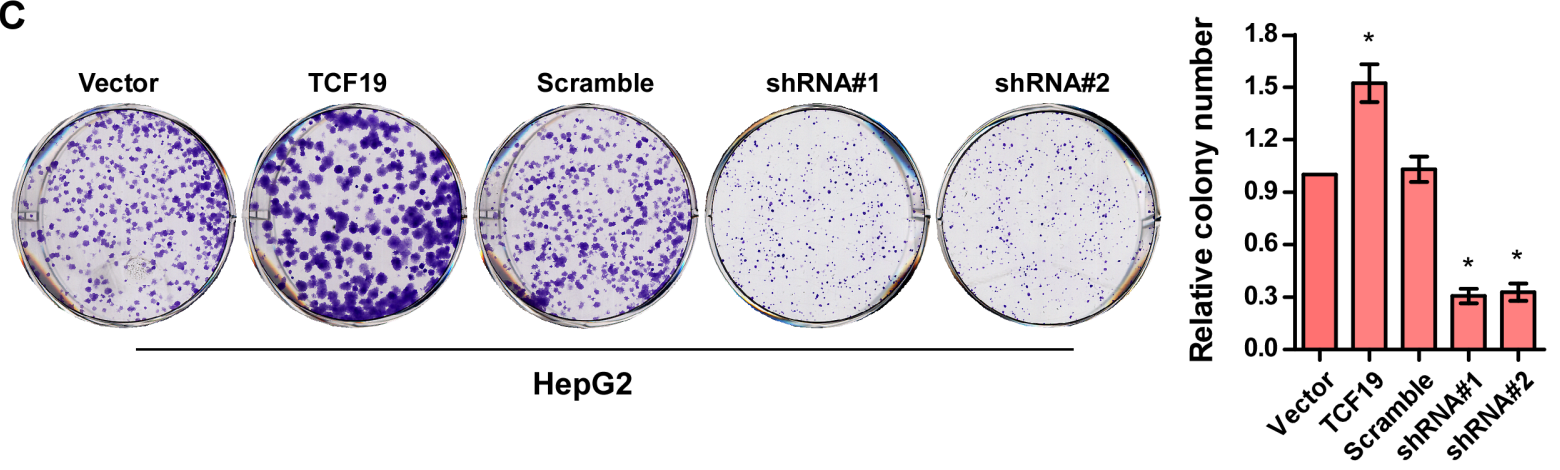

D

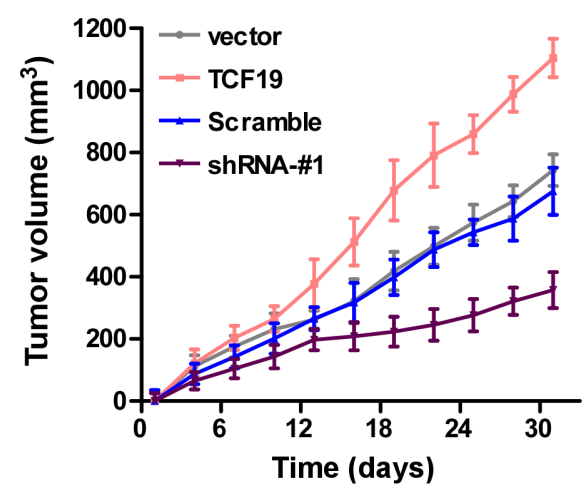

$\mathbf{E}$

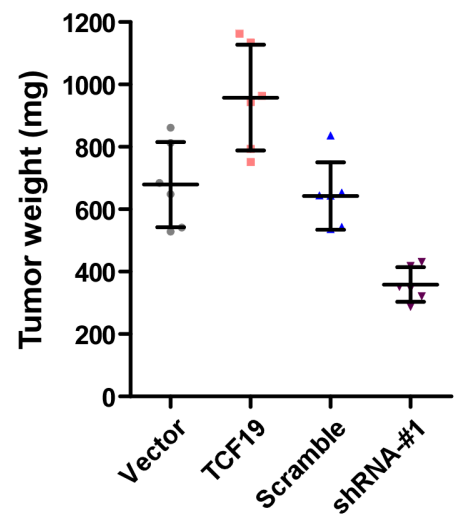

$\mathbf{F}$

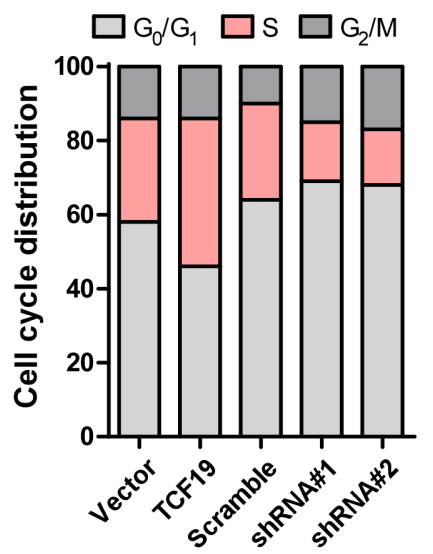

Figure 3. Overexpression of TCF 19 promotes proliferation of HCC cell. A) Western blotting assay of TCF19 in the indicated cells. $\alpha$-Tubulin is used as the loading control. B) Cell growth rate by MTT analysis in the indicated cells. C) The representative images (left panel) and colony numbers (right panel) of colony formation. D) Tumor volume growth curves injected with the indicated cells. E) Mean tumor weights. F) Cell cycle distribution in indicated cells. 

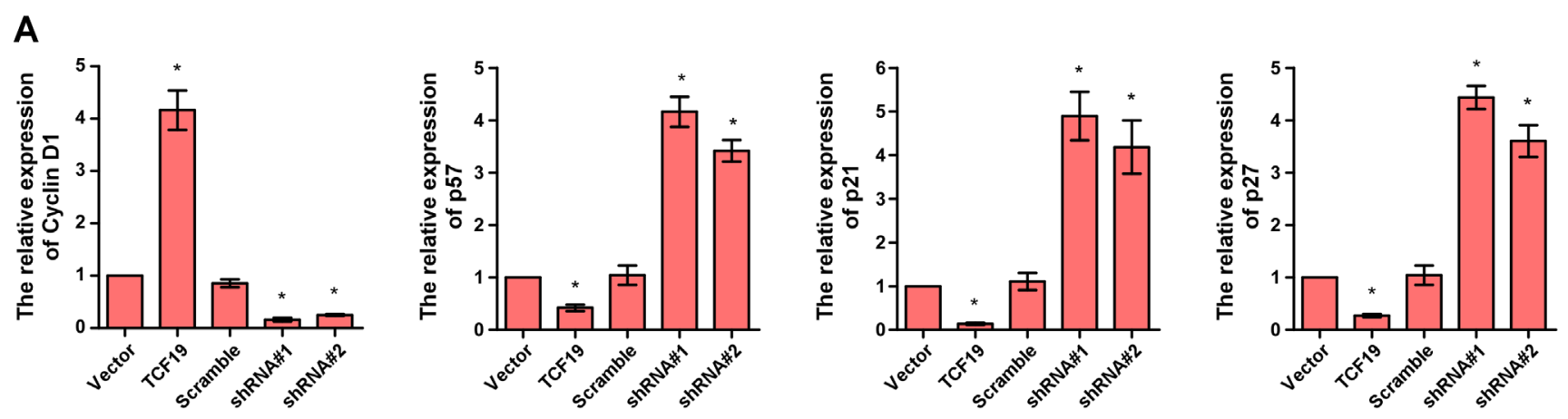

B

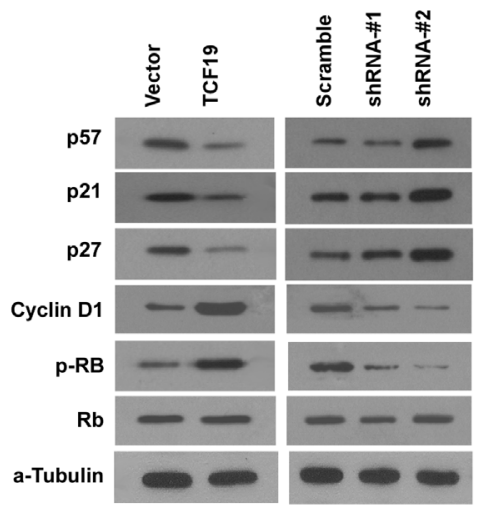

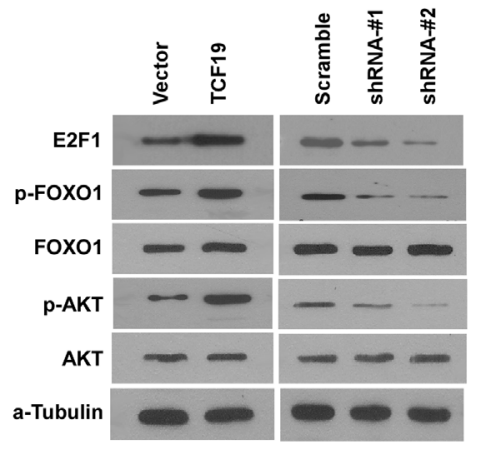

D

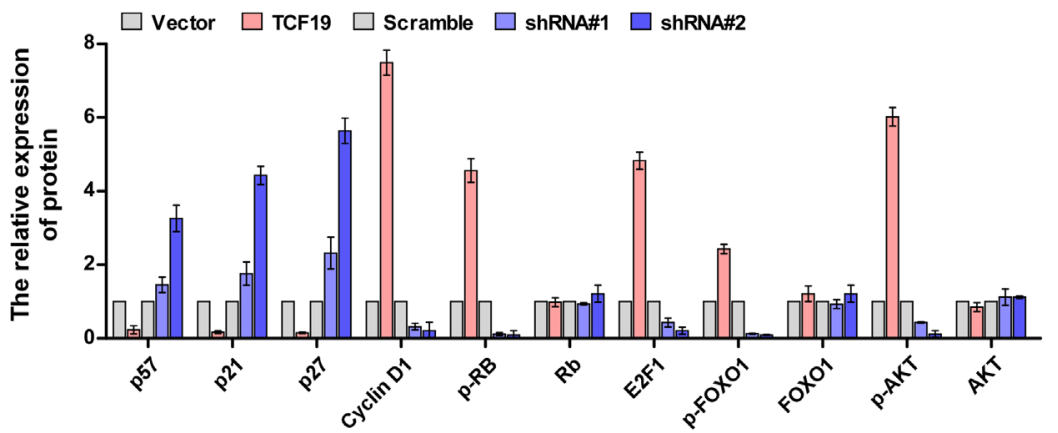

C
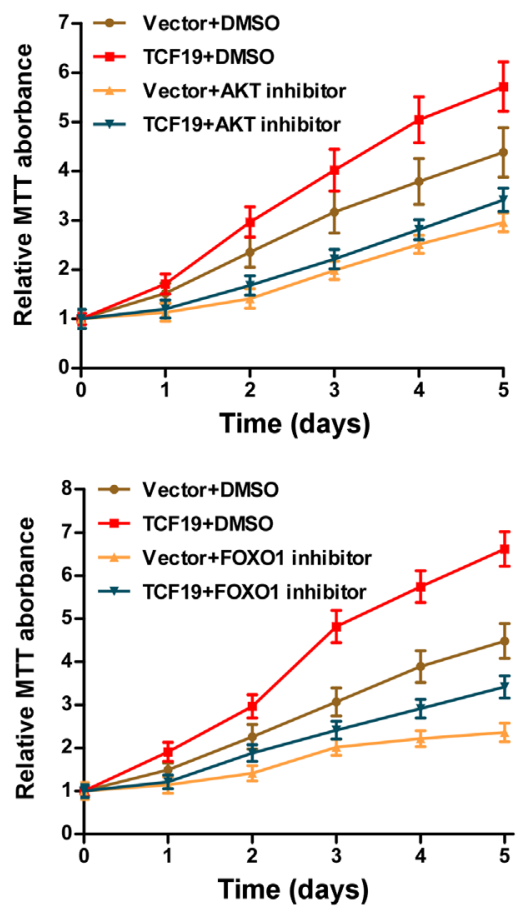

Figure 4. Over-expression of TCF19 promotes cell proliferation by activating the AKT/FOXO1 signaling pathway. A) The relative expression of cyclin $\mathrm{D} 1, \mathrm{p} 57^{\mathrm{Kip} 2}, \mathrm{p} 21^{\mathrm{Cip} 1}$ and $\mathrm{p} 27^{\mathrm{Kipl}}$ in the indicated cells. And mRNA level of interesting genes was normalized to that of $\alpha$-Tubulin. B) The western blotting

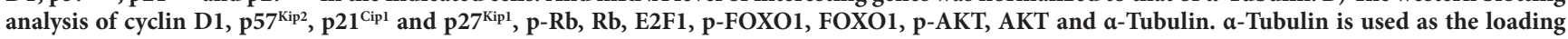
control. C) The cell growth rate of indicated cells treated by AKT (upper panel) and FOXO1 (lower panel) inhibitor by MTT assay. (D) The quantification analysis of indicated proteins.

ones formed by shRNA vector control cells (Figure 3E). Collectively, the over-expression of TCF19 enhances HCC cell proliferation and tumorigenesis.

We compared the cell cycle in HCC cells with normal level, over-expression andTCF19-silencing by flow cytometry. Figure 3F shows TCF19 up-regulation markedly increases the percentage of S-phase cells and decreases the percentage of cell in the $G_{0} / G_{1}$ phase. Conversely, inhibition of TCF19 shows the opposite effect. These results suggest that TCF19 contributes to the $\mathrm{G}_{1} / \mathrm{S}$ phase transition in HCC cells.
The combined results confirm that TCF19 elevates cell proliferation and tumorigenesis by enhancing $\mathrm{G}_{1} / \mathrm{S}$ phase transition.

Over-expression of TCF19 promotes HCC cell G1/S transition by activating AKT/FOXO1 signaling. To further clarify the mechanism by which over-expression of TCF19 enhances the proliferation of HCC cells we examined the effect of TCF19 on the expression of $\mathrm{p} 57^{\mathrm{KIP} 2}, \mathrm{p} 21^{\mathrm{Cip} 1}, \mathrm{p} 27^{\mathrm{Kip} 1}$ and cyclin D1 which are important cell cycle regulators. As shown in Figures $4 \mathrm{~A}, \mathrm{~B}$ and $\mathrm{D}$, the expression of $\mathrm{p} 57^{\mathrm{KIP} 2}$, 
$\mathrm{p} 21^{\mathrm{Cip} 1}$ and $\mathrm{p} 27^{\mathrm{Kip} 1}$ is significantly suppressed in TCF19-overexpressing cells and markedly increased in TCF19-silenced cells, at both the transcription and translation levels. In contrast, cyclin D1, p-Rb and E2F1 are increased in TCF19overexpressing cells and decreased in TCF19-silenced cells. This suggests that TCF19 contributes to the G1/S transition and enhances cell cycle progressionIt is well-known that cyclin D1, p57 Kip2 p2 $1^{\text {Cip1 }}$ and p27 $7^{\text {Kip1 }}$ are modulated by FOXO1 $[18,19]$. Hence, we further investigated the effect of TCF19 on the phosphorylation of FOXO1 and found that TCF19-overexpression significantly increases FOXO1 phosphorylation and TCF19-silence decreases it (Figures 4B and $4 \mathrm{D}$ ). FOXO1 is also downstream of AKT, so we assayed the level of p-AKT and discovered that AKT phosphorylation is elevated in TCF19 over-expression, but inhibited when TCF19 is silenced (Figures 4B and 4D). To further confirm the results, we used the $20 \mu \mathrm{M}$ AKT inhibitor to treat cells. (Perifosine; Selleck, Houston, Texas, USA) 10 nM FOXO1 inhibitor (AS1842856; Selleck, Houston, Texas, USA). MTT assay showed that AKT (Figure 4C upper panel) and the FOXO1 (Figure 4C lower panel) inhibitor eliminate the effect of TCF19 on cell proliferation. These results demonstrate that AKT/FOXO1 signaling activation is essential for stimulating TCF19 proliferation of HCC cells. Collectively, these results demonstrate that TCF19 promotes cell cycle progression by activating FOXO1 and AKT phosphorylation.

Clinical relevance of the TCF19/AKT/FOXO1 axis in HCC. To conclusively investigate whether there is correlation between TCF19 and the AKT/FOXO1 signaling axis, we examined the expression of TCF19 and p-AKT in 30 paraffinembedded, archived HCC tissues by IHC. The results demonstrated that the level of $\mathrm{p}-\mathrm{AKT}$ is significantly associated with the expression of TCF19 ( $p=0.001$, Figure 5 , Table $S 1)$, and the combined study results establish that TCF19 up-regulation activates AKT/FOXO1 signaling and leads to malignant progression in human hepatocellular carcinoma cells.

\section{Discussion}

The most important findings of the research are that TCF19 is significantly increased in HCC tissues and cell lines and that TCF19 over-expression promotes hepatocellular carcinoma cell tumorigenesis and proliferation. Further study showed that while TCF19 is considered to have some function in normal G1/S cell cycle transition [12], its overexpression promotes cell proliferation and tumorigenesis by accelerating this G1/S transition through FOXO1 and AKT phosphorylation.

Recent studies have shown that TCF19 has an important role in cell proliferation and is related to pancreatic $\beta$-cell mass, and TCF19 knockdown in the insulinoma cell line significantly reduces gene expression for the cell cycle and induces cell cycle arrest [17]. Our findings agree, and we showed that TCF19 is increased in HCC tissues and cell lines and TCF19 over-expression enhances HCC cell proliferation and tumorigenesis. Analysis of the TFC19 primary sequence then revealed that it contains a forkhead association (FHA) domain [20].

In this study, we found that TCF19 not only regulates FOXO1 phosphorylation, but it is also connected to proteins containing the FHA domain, including Ki67 [20, 21] which is a well-known marker of proliferation index [22]. These investigations confirmed our results that TCF19 is closely related with cell proliferation.
A

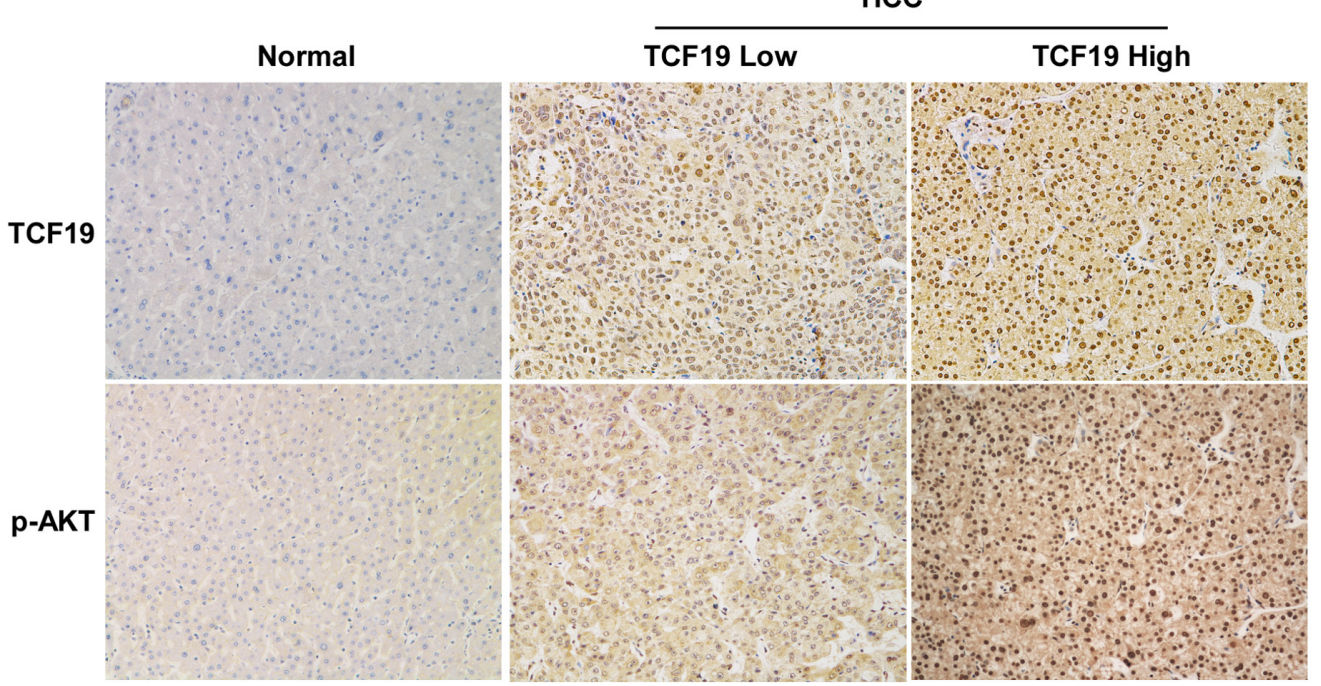

B

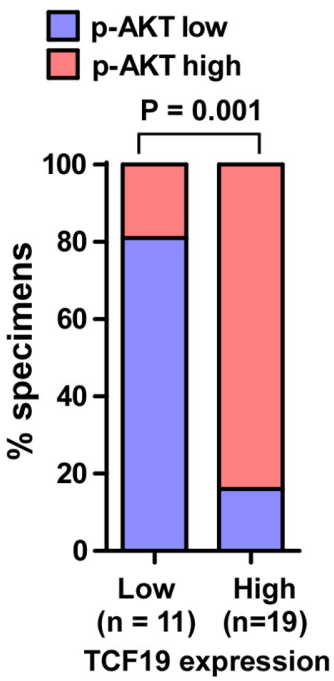

Figure 5. Clinical relevance of the TCF19/AKT/FOXO1 axis in HCC. A) The IHC assay of TCF19 and p-AKT in human normal liver and HCC tissues. B) The correlation analysis between TCF19 and p-AKT. 
TCF19 also contains a proline-rich region and PHD and Ring finger region [17], and this provides further evidence that TCF19 is very important in regulating the cell cycle. Hence, we found that up-regulation of TCF19 enhances the G1/S phase transition in HCC cells. Increasing studies have also established that FOXO1 is involved in diverse cellular pathways; including proliferation, stress resistance, differentiation and apoptosis [23]. Deregulation of FOXO1 has been found in many cancers, including prostate cancer, leukemia and melanoma [24-26]. Moreover, current knowledge shows that FOXO1 is regulated by $\mathrm{AKT}$ and $\mathrm{p}-\mathrm{AKT}$ can promote FOXO1 phosphorylation at Ser256, leading to FOXO1 translocation from nucleus to cytoplasm and inducing FOXO1 degradation by ubiquitination $[16,27,28]$. Our study demonstrated that over-expression of TCF19 can activate AKT and this then further stimulates TCF19 phosphorylation which shortens the cell cycle.

Our combined results show that TCF19 is increased in HCC cell lines and tissues and that TCF19 up-regulation induces cell cycle progression by simulating the AKT/ FOXO1 signaling pathway. We thereby provide correlation between TCF19 and HCC and suggest that TCF could be a successful new target in hepatocellular carcinoma treatment.

Supplementary information is available in the online version of the paper.

Acknowledgements: Guangdong Natural Science Foundation (No. 2015A030313756, 2018A0303130184); Science and Technology Programme of Guangzhou Municipal Government (No.201707010304), The Science foundation of Guangdong Second Provincial General Hospital (No. YY2016-007).

\section{References}

[1] YANG W, YAN HX, CHEN L, LIU Q, HE YQ et al. Wnt/ beta-catenin signaling contributes to activation of normal and tumorigenic liver progenitor cells. Cancer Res 2008; 68: 4287-4295. https://doi.org/10.1158/0008-5472.CAN-076691

[2] TORRE LA, BRAY F, SIEGEL RL, FERLAY J, LORTETTIEULENT J et al. Global cancer statistics, 2012. CA Cancer J Clin 2015; 65: 87-108. https://doi.org/10.3322/caac.21262

[3] KHEMLINA G, IKEDA S, KURZROCK R. The biology of Hepatocellular carcinoma: implications for genomic and immune therapies. Mol Cancer 2017; 16: 149. https://doi. org/10.1186/s12943-017-0712-x

[4] DUTTA R, MAHATO RI. Recent advances in hepatocellular carcinoma therapy. Pharmacol Ther 2017; 173: 106-117. https://doi.org/10.1016/j.pharmthera.2017.02.010

[5] HANAHAN D, WEINBERG RA. Hallmarks of cancer: the next generation. Cell 2011; 144: 646-674. https://doi. org/10.1016/j.cell.2011.02.013

[6] QIE S, DIEHL JA. Cyclin D1, cancer progression, and opportunities in cancer treatment. J Mol Med (Berl) 2016; 94: 1313-1326. https://doi.org/10.1007/s00109-016-1475-3
[7] SCHAAL C, PILLAI S, CHELLAPPAN SP. The Rb-E2F transcriptional regulatory pathway in tumor angiogenesis and metastasis. Adv Cancer Res 2014; 121: 147-182. https:// doi.org/10.1016/B978-0-12-800249-0.00004-4

[8] KITAJIMA S, TAKAHASHI C. Intersection of retinoblastoma tumor suppressor function, stem cells, metabolism, and inflammation. Cancer Sci 2017; 108: 1726-1731. https://doi. org/10.1111/cas.13312

[9] ATTWOOLL C, LAZZERINI DENCHI E, HELIN K. The E2F family: specific functions and overlapping interests. EMBO J 2004; 23: 4709-4716. https://doi.org/10.1038/ sj.emboj.7600481

[10] SINGH SK, BANERJEE S, ACOSTA EP, LILLARD JW, SINGH R. Resveratrol induces cell cycle arrest and apoptosis with docetaxel in prostate cancer cells via a p53/ p21WAF1/ CIP1 and p27KIP1 pathway. Oncotarget 2017; 8: 1721617228. https://doi.org/10.18632/oncotarget.15303

[11] Child ES, Mann DJ. The intricacies of p21 phosphorylation: protein/protein interactions, subcellular localization and stability. Cell Cycle 2006; 5: 1313-1319. https://doi.org/10.4161/ cc.5.12.2863

[12] KU DH, CHANG CD, KONIECKI J, CANNIZZARO LA, BOGHOSIAN-SELL L et al. A new growth-regulated complementary DNA with the sequence of a putative trans-activating factor. Cell Growth Differ 1991; 2: 179-186.

[13] MATSUURA K, ISOGAWA M, TANAKA Y. Host genetic variants influencing the clinical course of hepatitis B virus infection. J Med Virol 2016; 88: 371-379. https://doi. org/10.1002/jmv.24350

[14] MATHEW S, ABDEL-HAFIZ H, RAZA A, FATIMA K, QADRI I. Host nucleotide polymorphism in hepatitis B virus-associated hepatocellular carcinoma. World J Hepatol 2016; 8: 485-498. https://doi.org/10.4254/wjh.v8.i10.485

[15] YANG H, CHO ME, LI TW, PENG H, KO KS et al. MicroRNAs regulate methionine adenosyltransferase $1 \mathrm{~A}$ expression in hepatocellular carcinoma. J Clin Invest 2013; 123: 285298. https://doi.org/10.1172/JCI63861

[16] ZENG Z, LIN H, ZHAO X, LIU G, WANG X et al. Overexpression of GOLPH3 promotes proliferation and tumorigenicity in breast cancer via suppression of the FOXO1 transcription factor. Clin Cancer Res 2012; 18: 4059-4069. https://doi.org/10.1158/1078-0432.CCR-11-3156

[17] KRAUTKRAMER KA, LINNEMANN AK, FONTAINE DA, WHILLOCK AL, HARRIS TW et al. Tcf19 is a novel islet factor necessary for proliferation and survival in the INS-1 beta-cell line. Am J Physiol Endocrinol Metab 2013; 305: E600-610. https://doi.org/10.1152/ajpendo.00147.2013.

[18] BRUHL T, HEESCHEN C, AICHER A, JADIDI AS, HAENDELER J et al. p21Cip1 levels differentially regulate turnover of mature endothelial cells, endothelial progenitor cells, and in vivo neovascularization. Circ Res 2004; 94: 686692. https://doi.org/10.1161/01.RES.0000119922.71855.56

[19] ROEB W, BOYER A, CAVENEE WK, ARDEN KC. PAX3FOXO1 controls expression of the p57Kip2 cell-cycle regulator through degradation of EGR1. Proc Natl Acad Sci U S A 2007; 104: 18085-18090. https://doi.org/10.1073/ pnas.0708910104 
[20] LI J, LEE GI, VAN DOREN SR, WALKER JC. The FHA domain mediates phosphoprotein interactions. J Cell Sci 2000; 113 Pt 23: 4143-4149.

[21] DUROCHER D, HENCKEL J, FERSHT AR, JACKSON SP. The FHA domain is a modular phosphopeptide recognition motif. Mol Cell 1999; 4: 387-394. https://doi.org/10.1016/ S1097-2765(00)80340-8

[22] BOSCH DE, KILGORE MR, SCHMIDT RA, SWANSON $\mathrm{PE}, \mathrm{RENDI} \mathrm{MH}$ et al. Comparison of Proliferation Markers Ki67 and Phosphohistone-H3 (pHH3) in Breast Ductal Carcinoma In Situ. Appl Immunohistochem Mol Morphol 2017; 25:543-547.https://doi.org/10.1097/PAI.0000000000000555

[23] MURTAZA G, KHAN AK, RASHID R, MUNEER S, HASAN SMF et al. FOXO Transcriptional Factors and LongTerm Living. Oxid Med Cell Longev 2017; 2017: 3494289. https://doi.org/10.1155/2017/3494289
[24] ZHAO Y, TINDALL DJ, HUANG H. Modulation of androgen receptor by FOXA1 and FOXO1 factors in prostate cancer. Int J Biol Sci 2014; 10: 614-619. https://doi.org/10.7150/ ijbs. 8389

[25] ZHU H. Targeting forkhead box transcription factors FOXM1 and FOXO in leukemia (Review). Oncol Rep 2014; 32: 1327-1334. https://doi.org/10.3892/or.2014.3357

[26] TSITSIPATIS D, KLOTZ LO, STEINBRENNER H. Multifaceted functions of the forkhead box transcription factors FoxO1 and FoxO3 in skin. Biochim Biophys Acta 2017; 1861: 1057-1064. https://doi.org/10.1016/j.bbagen.2017.02.027

[27] ZHANG Y, GAN B, LIU D, PAIK JH. FoxO family members in cancer. Cancer Biol Ther 2011; 12: 253-259.

[28] LIN C, WU Z, LIN X, YU C, SHI T et al. Knockdown of FLOT1 impairs cell proliferation and tumorigenicity in breast cancer through upregulation of FOXO3a. Clin Cancer Res 2011; 17: 3089-3099. https://doi.org/10.1158/1078-0432. CCR-10-3068 


\section{TCF19 enhances cell proliferation in hepatocellular carcinoma by activating the ATK/FOXO1 signaling pathway}

C. X. ZENG ${ }^{1}$, S. B. FU' ${ }^{2}$, W. S. FENG', J. Y. ZHAO ${ }^{1}$, F. X. $\mathrm{LI}^{1}$, P. GAO ${ }^{1, \star}$

Supplemental Material

\begin{tabular}{|c|c|c|}
\hline \multirow[b]{2}{*}{ Characteristics } & \multicolumn{2}{|c|}{ TCF19 expression } \\
\hline & $\begin{array}{c}\text { Low, } \\
\text { no. cases }\end{array}$ & $\begin{array}{l}\text { High, } \\
\text { no. cases }\end{array}$ \\
\hline \multicolumn{3}{|l|}{ Age (years) } \\
\hline$<50$ & 5 & 8 \\
\hline$\geq 50$ & 6 & 11 \\
\hline \multicolumn{3}{|l|}{ Clinical stage } \\
\hline I-II & 4 & 7 \\
\hline III-IV & 7 & 12 \\
\hline \multicolumn{3}{|l|}{ Histologic grade } \\
\hline High/moderate & 3 & 13 \\
\hline Poor & 8 & 6 \\
\hline \multicolumn{3}{|c|}{ Recurrent status (at follow-up) } \\
\hline Negative & 11 & 18 \\
\hline Positive & 0 & 1 \\
\hline \multicolumn{3}{|c|}{ Alpha fetal protein (AFP) } \\
\hline$\leq 400 \mu \mathrm{g} / \mathrm{ul}$ & 4 & 2 \\
\hline$>400 \mu \mathrm{g} / \mathrm{ul}$ & 7 & 17 \\
\hline \multicolumn{3}{|l|}{ Tumor size } \\
\hline$\leq 5 \mathrm{~cm}$ & 6 & 5 \\
\hline$>5 \mathrm{~cm}$ & 5 & 14 \\
\hline \multicolumn{3}{|l|}{ p-AKT } \\
\hline Low & 9 & 3 \\
\hline High & 2 & 16 \\
\hline
\end{tabular}

